\title{
Aproximación a los cambios intra-anuales en el contenido de energía del ovario de sardina (Sardinops sagax Jenyns, 1842) en el norte de Chile
}

\author{
Renzo Tascheri O. ${ }^{1}$ y Gabriel Claramunt Q. ${ }^{2}$ \\ ${ }^{1}$ Instituto de Fomento Pesquero, Casilla 8-V, Valparaíso, Chile \\ ${ }^{2}$ Universidad Arturo Prat, Casilla 121, Iquique, Chile
}

Resumen. Se estimó el peso seco del ovario y de las dos modas de ovocitos vitelados de hembras maduras de sardinas de dos estratos de talla (300 hembras de $26-28 \mathrm{~cm}$ y 327 hembras de $28-30 \mathrm{~cm}$ ), durante el ciclo reproductivo de abril'92-marzo' 93 en el norte de Chile ( $\left.19^{\circ}-22^{\circ} \mathrm{S}\right)$. Las estimaciones se obtuvieron mediante la función Weibull, relacionando el diámetro de los ovocitos y su peso seco, conocidos el peso húmedo del ovario, el peso húmedo de la submuestra de tejido ovárico para el cálculo de la fecundidad parcial y la distribución de frecuencias del diámetro de los ovocitos contenidos en la submuestra. Las variables estimadas fueron relacionadas con estimaciones del mismo ciclo reproductivo de fecundidad, tamaño medio del ovocito de la moda más avanzada, índice de condición, frecuencia de desove y porcentaje de hembras desovantes.

El peso seco del ovario de las modas de ovocitos vitelados siguieron la misma tendencia que el tamaño medio de ovocitos de la moda más avanzada y la fecundidad, es decir con valores altos en los dos períodos de mayor incidencia de desove (invierno y verano), y bajos en períodos de reducida actividad sexual. Una baja en la fecundidad y tamaño medio del ovocito de la moda más avanzada en julio y en las variables estimadas, ocurre junto a una baja en el índice de condición en ambos estratos de talla, discutiéndose su posible relación con el evento El Niño 1991-92.

Palabras clave: energía reproductiva, peso seco, ovocitos, vitelo, ovario, sardina, norte de Chile.

\section{An aproach to intra-anual changes in the energy contents of the ovary of south american pilchard (Sardinops sagax Jenyns, 1842) in northern Chile}

\begin{abstract}
Ovary and yolked oocytes modal dry weight of mature females of south American pilchard, distributed in two total length strata (300 females of $26-28 \mathrm{~cm}$ and 327 of $28-30 \mathrm{~cm}$ ), was estimated on a monthly basis in northern Chile $\left(19^{\circ}-22^{\circ} \mathrm{S}\right)$ during the reproductive cycle (between april' 92 and march ' 93 ). The estimates were obtained using a Weibull function which relates the oocyte diameter to its dry weight, given the wet weight of the ovary, the wet weight of the subsample of ovarian tissue for the fecundity estimates and the oocyte diameter frequency distributions. The estimated variables fluctuations were related to monthly estimates of fecundity, oocyte average size for the more advanced batch, condition index, spawning frequency and the fraction of spawning females for the same reproductive cycle.

The estimated ovary and yolked oocytes modal dry weight followed the same trend as the asymptotic oocyte dry weight and batch fecundity, with high values for the two spawning periods (winter and summer) and low values for the periods of less reproductive activity. A low fecundity and asymptotic oocyte dry weight value occurred in july along with a low value in the condition index by length stratum. A possible related effect to El Niño phenomenon is discussed.
\end{abstract}

Key words: reproductive energy, oocyte, dry weight, yolk, ovary, pilchard, northern Chile. 


\section{INTRODUCCION}

En general, los cambios de una población dependen esencialmente de la reproducción, crecimiento y mortalidad. Los dos últimos procesos se manifiestan con mayor intensidad en los estados tempranos del desarrollo, por la cual esta etapa de la vida de los peces es reconocida como relevante en biología pesquera.

La variabilidad del tamaño del huevo puede ser un factor importante en la sobrevivencia de los estados tempranos de los peces. Numerosos autores (Blaxter y Hempel, 1963; Knudsen y Tilseth, 1985), han propuesto que el tamaño del huevo (diámetro, peso seco) influye en la sobrevivencia larval por sus efectos en el tamaño de la larva, tasas de crecimiento y actividad. Huevos más grandes proveen más energía para el desarrollo y crecimiento (Hempel, 1979) y, en general, producen larvas más grandes que son capaces de evadir a los predadores en forma más efectiva (Miller et al., 1988), sobrevivir más sin alimentarse (Hunter, 1981), abarcar un mayor volumen de agua en busca de su presa (Webb y Weihs, 1986), y comer presas de mayor rango de tamaño (Hunter, 1981).

En relación a la fecundidad, Cushing (1990) señala que con una menor abundancia de huevos y/o fecundidad hay una mayor probabilidad de que ocurra un desfase con el ciclo de producción que a una abundancia mayor; es decir, el reclutamiento es usualmente más variable con una abundancia menor. Tanto el tamaño del huevo como la fecundidad varían estacionalmente (Claramunt et al., 1994; Le Clus, 1992; Macewicz y Hunter, 1993), y están frecuentemente relacionados de forma inversa (Blaxter y Hunter, 1982). Esto se ha interpretado como una evidencia de la conexión entre la sobrevivencia durante los estados tempranos de la vida y las variables reproductivas (Bradford y Stephenson, 1992), ya que esta relación aumentaría la sobrevivencia frente a condiciones biológicas y oceanográficas variables.

Según Ware (1975), parece entonces que la estrategia de reproducción puede ser interpretada como un compromiso entre peso y número, de modo que: a) las nuevas larvas eclosionadas sean del tamaño apropiado para alimentarse de la población zooplanctónica imperante; y b) de modo que suficientes individuos sobrevivan en promedio, dentro de los límites de la provisión de alimento imperante, para asegurar un reemplazo óptimo de la población adulta.

La sardina presenta un ovario asincrónico con desove fraccionado y mezcla de clases anuales, observándose en un mismo período tanto ejemplares reproduciéndose como otros en reposo. Sin embargo, por lo general predomina un estado de madurez determinado, visualizándose en la zona AricaAntofagasta en los períodos enero-marzo (desove secundario) y junio-octubre (desove principal) la mayor proporción de hembras maduras (Martínez et al., 1990). También ha sido descrito un período de reposo sexual entre abril y mayo, observándose una disminución del peso de los ovarios producto de la liberación de los ovocitos maduros y su reabsorción (Martínez et al., 1990).

En la sardina también ha sido observada una tendencia a la fecundidad parcial, siendo este contraste evidente entre los períodos reproductivos de invierno y verano (Claramunt et al., 1994). Sin embargo, esta no es una relación funcional inversa; en otras palabras, los cambios en la fecundidad parcial no son compensados por los cambios en el tamaño del ovocito como ha sido sugerido por Alheit (1988) para Sprattus sprattus; esto es debido a que el peso del ovario no es constante a través de un mismo período reproductivo (Claramunt et al., 1994). Por otro lado, diversos autores han relacionado la variación en el tamaño del huevo con variaciones en temperatura, encontrando relaciones inversas (Ware, 1997; Le Clus, 1979; Imai y Tanaka, 1987; De Martini, 1991).

En la sardina Claramunt et al. (1994) observan una disminución en el tamaño del ovocito entre invierno y verano; pero al considerar sólo el período de verano esta aparente tendencia a disminuir su tamaño con la temperatura no fue observable, incluso se constató la tendencia opuesta. Estas variaciones del tamaño y número de ovocitos producidos las atribuyen a diferencias estacionales en la canalización de la energía, lo que implica algún patrón de fluctuación en la cantidad de energía destinada a reproducción que explica las variaciones observadas. Tal fluctuación es causada en gran medida por cambios en la fecundidad, en el peso de los ovocitos y en el valor calórico (cantidad de energía por unidad de masa, cal $/ \mathrm{mg}$ ) del material seco de los ovocitos (Kamler, 1992). Por otra parte, el ta- 
maño de los ovocitos en el ovario está determinado por su contenido de vitelo (Wallace y Selman, 1981; Dumont y Brummett, 1985), el que se puede cuantificar indirectamente a través del peso seco del huevo (Le Clus, 1979).

Debido a su gran concentración de lípidos, la energía en huevos de peces es particularmente alta (Wooton, 1979 fide Kamler, 1992), a su vez la cantidad de un componente químico en un huevo es determinada por dos factores: el tamaño del huevo y la concentración del componente. Kamler (1992) muestra que el valor calórico de los huevos varía en un factor de dos aproximadamente, el que es comparativamente menor a su variabilidad en peso de varias órdenes de magnitud. Consecuentemente, si bien el valor calórico contribuye a la variabilidad en el contenido energético de un huevo, lo haría en menor grado que su peso.

En el presente trabajo se asume que el rango de variación en el valor calórico del huevo de sardina es menor al rango de variación de su peso seco; por lo tanto, el peso del huevo sería una buena aproximación de su contenido de energía.

Los factores más frecuentemente citados como modificadores de la fecundidad (Tsuruta y Hirose, 1989), tamaño del ovocito (Clarke, 1989), tasa metabólica (Kamler, 1992), tasas de crecimiento (Daoulas y Economou, 1986), actividad hormonal (Billard et al., 1982) y duración e intensidad del desove (Kjesbu, 1989), son la temperatura y la disponibilidad de alimento. Luego se determinaron las fluctuaciones estacionales en el peso seco de la gónada y las modas de ovocitos vitelados como una aproximación al patrón de fluctuación intra-anual de la energía destinada a reproducción durante el período de estudio, analizando a la vez posibles relaciones de estas fluctuaciones con la temperatura y condición de los desovadores.

Finalmente, los análisis se conducen por separado en dos estratos de tallas, los que corresponden a los de mayor talla de los tres utilizados por Claramunt et al. (1994). Esta estratificación considera que las hembras de mayor tamaño poseen gónadas de mayor peso (Blaxter y Hunter, 1982), y períodos de desove más intensos (Claramunt et al., 1994) y extensos (Pizarro, 1990), por lo que parece razonable que los patrones de fluctuación mensual en el material seco del ovario sean también dependientes de la talla.

\section{MATERIALES Y METODOS}

La información utilizada corresponde a los datos obtenidos por Claramunt et al. (1994), entre abril de 1992 y marzo de 1993, y comprende la longitud total de los individuos de sardina (precisión 0,1 cm), peso total de los ejemplares $(0,1 \mathrm{~g})$, peso húmedo del ovario $(0,01 \mathrm{~g})$, estados de madurez sexual, distribuciones de frecuencia del diámetro de los ovocitos, peso seco promedio por diámetro del ovocito, fecundidad parcial y diámetro medio de la moda más avanzada de ovocitos. Una descripción del muestreo y de la metodología se encuentra en Claramunt et al. (1994); sin embargo, determinados aspectos relevantes para este estudio se describen a continuación.

A los ovarios frescos obtenidos para el estudio de fecundidad parcial se les extrajo una submuestra de tejido, la que fue pesada con precisión $0,0001 \mathrm{~g}$ y preservada en solución Gilson modificada por Simpson (1951). Los ovocitos una vez disgregados del estroma se separaron por clases de tamaño, a través de una batería de tamices con un rango de 200 a $1000 \mu \mathrm{m}$ a intervalos de malla de $50 \mu \mathrm{m}$, con arrastre en agua en un sistema de vibración. La malla de 200 micrones representó el límite natural de los ovocitos considerados, ya que próximo a ese tamaño se inicia el depósito del vitelo en el citoplasma (Herrera y Claramunt, 1990). El conteo bajo lupa estereoscópica de los ovocitos retenidos en cada malla da como resultado una distribución de frecuencias de diámetro.

Las hembras analizadas se seleccionaron por talla, considerando como criterio los ejemplares de 26 a $28 \mathrm{~cm}$ y 28 a $30 \mathrm{~cm}$ de longitud total, los que fueron analizados por separado. Esta estratificación considera que las hembras de mayor tamaño poseen gónadas de mayor peso y poseen períodos de desove más intensos y extensos. La selección de este rango de talla se debe a la escasa ocurrencia en la muestra de ejemplares maduros de sardina con longitud total menor a $26 \mathrm{~cm}$. Para evitar fluctuaciones en el peso del ovario, debido al proceso natural del desarrollo de los ovocitos, sólo se consideraron aquellas hembras en estado de madurez próximo a la hidratación, según lo diagnosticado por Claramunt et al. (1994) a través de criterios histológicos, de las cuales se hubiesen efectuado análisis de fecundidad parcial. De acuerdo a lo anterior se seleccionó un total de 627 hembras, de las cuales 300 de 26 
a $28 \mathrm{~cm}$ (Estrato A) y 327 de 28 a $30 \mathrm{~cm}$ (Estrato B); el número de ejemplares obtenidos mensualmente se presenta en la Tabla 1.

Tabla 1. Sardinas hembras analizadas por estratos de talla (A: 26-28 cm y B: $28-30 \mathrm{~cm}$ ) durante 1992-93.

H: histología; F: fecundidad.

Table 1: Sardine females analized by lenght stratum (A: 26-28 cm and B: $28-30 \mathrm{~cm}$ ) during 1992-93.

H: histology; F: fecundity.

\begin{tabular}{|l|rr|rl|}
\hline & \multicolumn{2}{|c|}{ Estrato A } & \multicolumn{2}{c|}{ Estrato B } \\
Mes & H & F & H & F \\
\hline Abr & 25 & 22 & 37 & 20 \\
May & 28 & 19 & 23 & 14 \\
Jun & 33 & 33 & 29 & 26 \\
Jul & 24 & 17 & 14 & 10 \\
Ago & 33 & 23 & 31 & 21 \\
Sep & 29 & 27 & 31 & 22 \\
Oct & 24 & 23 & 29 & 26 \\
Nov & 7 & 7 & 15 & 13 \\
Dic & 38 & 38 & 34 & 32 \\
Ene & 7 & 7 & 28 & 26 \\
Feb & 27 & 27 & 29 & 28 \\
Mar & 25 & 24 & 27 & 26 \\
\hline & 300 & 267 & 327 & 264 \\
\hline
\end{tabular}

Se obtuvo estimaciones del peso seco de la moda intermedia de ovocitos (PSMI; moda de ovocitos en desarrollo inmediatamente anterior a la última moda) y de la última moda (PSUM; moda más avanzada de ovocitos vitelados), a partir de la conversión a peso seco de cada una de las distribuciones de frecuencias de diámetro de ovocitos generadas durante los estudios de fecundidad parcial, considerando la siguiente ecuación:

$$
P S M=\sum_{i=1}^{c} f_{i} \hat{P}_{i}
$$

donde: PSM = peso seco de la moda de ovocitos vitelados; $\mathrm{c}=$ número de clases de diámetro; $f_{i}=$ frecuencia observada de ovocitos de diámetro $\mathrm{D}_{\mathrm{i}}$; $\mathrm{y}$ $\mathrm{P}_{\mathrm{i}}=$ peso seco estimado de ovocitos de diámetro $\mathrm{D}_{\mathrm{i}}$ estimado en la ecuación (2).

El peso seco estimado se obtuvo por medio de la función Weibull (1951) (Ecuación 2), utilizando datos de 45 hembras seleccionadas mensualmente sobre la base de un estado avanzado de madurez ovárica. A éstas se les cuantificó los ovocitos rete- nidos en cada tamiz, observando éste con una lupa estereoscópica y utilizando un contador manual; luego los ovocitos se disecaron en una estufa a $60^{\circ} \mathrm{C}$ por 24 horas, después a temperatura ambiente por un lapso no inferior a 24 horas (Le Clus, 1979), y finalmente se les consignó el peso $(0,1 \mathrm{mg}$ precisión). Con el número y el peso se estimó el peso seco promedio por diámetro de ovocito del siguiente modo:

$$
P_{\mathrm{i}}=K\left(1-e^{-\left(D_{i} / S\right)^{b}}\right)
$$

donde: $\mathrm{P}_{\mathrm{i}}=$ peso seco de los ovocitos $(\mathrm{mg}) ; \mathrm{D}_{\mathrm{i}}=$ diámetro de los ovocitos $(\mu \mathrm{m}) ; \mathrm{K}=$ peso seco asintótico de los ovocitos; $\mathrm{S}=$ diámetro en que el peso seco es $0,63 \mathrm{~K} ; \mathrm{y} \mathrm{b}=$ factor de forma.

El ajuste no lineal se realizó por medio del algoritmo de Marquardt (1963), utilizando el programa computacional estadístico Statgraphics (Manugistics, 1992). Las estimaciones iniciales de los parámetros se obtuvieron de los valores reportados para abril por Claramunt et al. (1994), utilizando subsecuentemente para los meses que siguen el estimado para el mes anterior. La dependencia de los valores estimados de las variables PSUM y PSMI del peso húmedo de la sub-muestra de tejido ovárico del cual se obtuvieron las distribuciones de frecuencias de diámetro utilizadas en su cálculo, fue anulada mediante la estandarización de los valores a $1 \mathrm{~g}$ de peso húmedo (peso seco relativo). Según lo anterior, el peso seco de la submuestra del tejido ovárico se obtiene de la sumatoria de las estimaciones de peso seco de las dos modas de ovocitos vitelados presentes en las distribuciones de frecuencia de los peces seleccionados. Dado que se conoce el peso húmedo de la submuestra como de la gónada completa, mediante un cálculo simple se tiene una estimación del peso seco de esta última (PSG). En la conversión a peso seco de las distribuciones de frecuencia de diámetro se utilizó una función mensual para ambos estratos de talla, pues la talla no tiene influencia sobre la cantidad de vitelo aportada al huevo (Claramunt et al., 1994). El valor del parámetro $\mathrm{K}$ de esta función se considera como un índice adecuado de la variación estacional del tamaño del ovocito durante el período de estudio.

La hipótesis de ninguna diferencia entre meses para los valores promedio estimados de PSUM, PSMI y PSG, se puso a prueba para el estrato de talla B por medio del análisis de varianza, luego de constatar mediante una prueba de bondad de ajuste 
de Kolmogorov-Smirnov (Conover, 1980) que los valores mensuales estimados de estas variables se distribuyen normalmente. Se utilizó la diferencia mínima significante (Steel y Torrie, 1985) para efectuar comparaciones planeadas entre abril y mayo (comparaciones intra-períodos deprimidos), entre junio, julio, agosto y septiembre, entre diciembre, enero y febrero (comparaciones intra-períodos de máxima actividad reproductiva), y entre junio y febrero. Estos últimos reportados por Claramunt et al. (1994) como meses de alto valor de peso seco asintótico y fecundidad parcial para el ciclo reproductivo 1992-93 (comparaciones inter-períodos de máxima actividad). La hipótesis de ninguna diferencia entre los valores promedio mensuales estimados de PSUM, PSMI y PSG para el estrato de talla A se investigó mediante una prueba de KruskalWallis (Zar, 1984), debido al pequeño número de datos que se obtuvo para este estrato durante noviembre de 1992 y enero de 1993 (Tabla 1), y a que los valores estimados de PSUM para abril no se distribuyeron normalmente $(\mathrm{K}-\mathrm{S}=0,27 ; \mathrm{n}=25 ; \mathrm{a}=$ $0,05)$. Las comparaciones no paramétricas múltiples se realizaron por medio del método de Nemenyi (1963), modificado por Wilcoxon y Wilcox (1964) fide Zar (1984), para el caso de pares de medias de igual número de datos y por medio del método de Dunn (1964), modificado por Hollander y Wolfe (1973) fide Zar (1984), para pares de medias con números desiguales de datos.

Para el estudio de la condición de los peces se utilizó la metodología propuesta por Patterson (1992), por medio de la cual todos los factores considerados y relacionados a la condición del pez son incluidos directamente en un análisis de regresión (Ecuación 3); luego se puede estudiar la significancia de incluir estas variables por medio de métodos estadísticos convencionales. Se consideraron inicialmente como variables explicatorias, además de la longitud total (L), el diámetro medio de la moda más avanzada (DOMA), la fecundidad parcial, PSUM, PSMI y PSG. La elección del mejor conjunto de variables explicatorias se realizó por medio del ajuste de todas las posibles regresiones para ambos estratos y descartando los modelos inapropiados por medio de los criterios Cp y el cuadrado medio del error (Canavos, 1987; Johnson y Wichern, 1988).

$$
\ln W_{\mathrm{i}}=\beta_{0}+\beta_{1} \cdot \ln L_{1}+\sum_{j=2}^{Z} \beta_{\mathrm{j}} \cdot x_{\mathrm{ji}}+e_{\mathrm{i}}
$$

donde: $\mathrm{W}_{\mathrm{i}}=$ peso corporal observado del pez-iésimo; $\beta_{0}=$ intercepto; $\beta_{1}=$ coeficiente de $1 \mathrm{~nL}_{\mathrm{i}} ; \mathrm{L}_{\mathrm{i}}=$ longitud observada del pez-iésimo; $\mathrm{z}=$ número de variables explicatorias; $\beta_{\mathrm{j}}=$ coeficiente de la variable explicatoria $\mathrm{j} ; \mathrm{x}_{\mathrm{ij}}=$ variable explicatoria $\mathrm{j}$ observada para el pez-iésimo; $\mathrm{e}_{\mathrm{i}}=$ error aleatorio $\sim \mathrm{N}\left(0, \delta^{2}\right)$; e $\mathrm{i}=1, \mathrm{~N}$ (número total de datos para el ciclo anual).

Para el estudio de posible colinealidad entre las variables se analizó la matriz de correlación y se calculó el índice de condicionamiento (Canavos, 1987). Se efectuaron pruebas de significancia estadística para los modelos ajustados, como también para cada uno de los parámetros estimados (Zar, 1984), y se probó la validez de los modelos ajustados por medio de un análisis de los respectivos residuos (Canavos, 1987; Johnson, y Wichern, 1988).

Se calcularon los coeficientes beta con el fin de efectuar afirmaciones sobre la importancia relativa de las variables independientes incluidas en el modelo de regresión múltiple (Pindyck y Rubinfeld, 1980). La variación estacional en la condición se investigó mediante la inclusión de su variación mensual en el modelo (Patterson, 1992; Charterjee y Price, 1977) (Ecuación 4):

$\ln W_{\mathrm{i}}=\beta_{0}+\beta_{1} \cdot \ln L_{1}+\sum_{j=2}^{z} \beta_{\mathrm{j}} \cdot x_{\mathrm{ji}}+\sum_{k=1}^{11} \sum_{l=1}^{\mathrm{m}_{\mathrm{k}}} \gamma_{\mathrm{k}} \cdot l_{\mathrm{i}}+e_{\mathrm{i}}$

donde: $\mathrm{W}_{\mathrm{i}}=$ peso corporal observado del pez-iésimo; $\beta_{0}=$ intercepto; $\beta_{1}=$ coeficiente de $1 \mathrm{~nL}_{\mathrm{i}} \mathrm{L}_{\mathrm{i}}=$ longitud observada del pez-iésimo; $\mathrm{z}=$ número de variables explicatorias; $\beta_{\mathrm{j}}=$ coeficiente de la variable explicatorio $\mathrm{j} ; \mathrm{x}_{\mathrm{ij}}=$ variable explicatoria $\mathrm{j}$ observada para el pez-iésimo; $\gamma_{k}=$ factores de estacionalidad mensual; $\mathrm{m}_{\mathrm{k}}=$ número de datos del mes $\mathrm{k} ; \mathrm{l}_{\mathrm{i}}=$ registro número 1 del pez-iésimo para el mes $\mathrm{k}$; $\mathrm{e}_{\mathrm{i}}=$ error aleatorio $\sim \mathrm{N}\left(0, \delta^{2}\right)$; e $\mathrm{i}=1, \mathrm{~N}$ (número total de datos para el ciclo anual).

\section{RESULTADOS}

Los parámetros de las funciones Weibull estimadas para cada mes se muestran en la Tabla 2. En todos los meses considerados los parámetros de los modelos obtenidos fueron significativamente distintos de cero. Los valores mensuales del peso asintótico alcanzado por los ovocitos se muestran en la Fig. 1. 


\section{Peso seco de la última moda}

Para las 300 hembras correspondientes al estrato de talla A (Tabla 1), el peso seco medio total de la última moda fue de 200,33 mg (Fig. 2a), con una desviación estándar de $60,82 \mathrm{mg}$, fluctuando entre 167,31 y $250,64 \mathrm{mg}( \pm 64,82$ y $\pm 63,18 \mathrm{mg}$ respectivamente). Para las 327 hembras seleccionadas para el estrato de talla B (Tabla 1), la medida general fue de 199,06 $\pm 58,09 \mathrm{mg}$ (Fig. 2b), con un mínimo de $141,22 \pm 42,14 \mathrm{mg}$ y un máximo de 242,62 $\pm 60,24$ mg. Las diferencias entre meses para los valores estimados promedio de PSUM, fueron significativas para ambos estratos de talla $(\mathrm{p}<0,05)$.

\section{Peso seco de la moda intermedia}

El peso seco estimado de la moda intermedia tuvo un promedio total para el estrato de talla A de 131,07 mg (Fig. 2a), con una desviación estándar de $\pm 58,95$ mg y promedios mensuales máximos y mínimos de $184,29 \pm 50,59$ y $66,88 \pm 23,79 \mathrm{mg}$ respectivamente. Para el estrato de talla B se estimó un promedio total de 125,11 $\pm 54,61 \mathrm{mg}$ (Fig. 2b), con un máximo de $169,45 \pm 54,92 \mathrm{mg}$ y un mínimo de 67,05 $\pm 27,14 \mathrm{mg}$.

Fluctuación mensual del peso seco de las modas por estrato de talla

Tanto el peso seco de la última moda como de la moda intermedia presentan, en general, para ambos estratos de talla, valores bajos para los períodos deprimidos reproductivamente (abril, mayo y noviembre), coincidiendo con bajos valores del tamaño del ovocito y de fecundidad. En tanto que los valores altos para los períodos de máxima actividad reproductiva coinciden con altos valores de tamaño del ovocito y fecundidad (junio y febrero), encontrándose diferencias significativas entre los meses correspondientes a ambos períodos $(\mathrm{p}<0,05)$ (Fig. 1, 2 y 3). No obstante, cuando se compara junio (mes de mayor valor de PSUM y PSMI para el desove de invierno) con febrero (máximo valor de estas variables durante el desove de verano en los dos estratos de talla considerados) no se encuentra ninguna diferencia $(\mathrm{p}>0,05)$.

Una prueba de diferencias de medias entre estratos de talla para junio, enero, abril, noviembre y julio (meses de valores máximos y mínimos de PSUM y PSMI; julio se comparó por aparecer como una situación excepcional), indica que para el ciclo

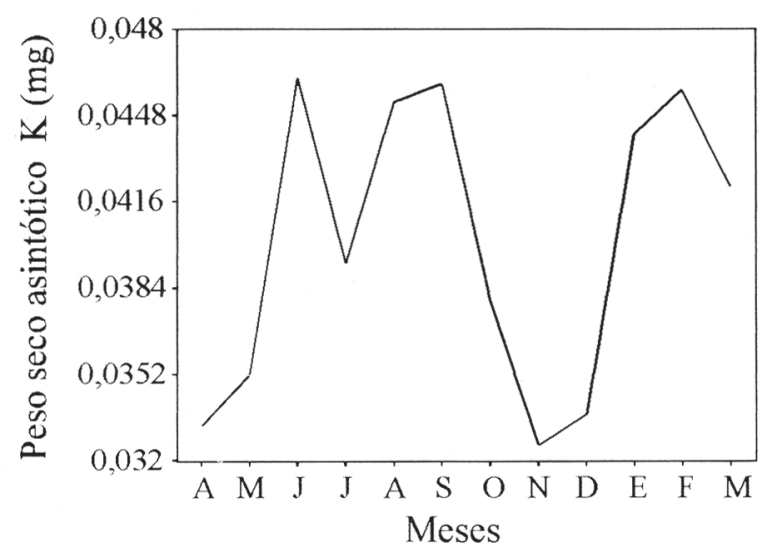

Figura 1. Peso seco asintótico (K) de ovocitos de sardina correspondiente a funciones Weibull ajustadas mensualmente durante el ciclo reproductivo 1992-93.

Figure 1. Asymptotic dry weight (K) of sardine oocytes corresponding to Weibull functions monthly adjust during the 1992-93 reproductive cycle.

Tabla 2. Parámetros de funciones Weibull ajustadas entre el diámetro y el peso seco de los ovocitos de sardina (1992-93).

Table 2. Estimated parameters of Weibull functions adjust between the oocyte diameter and its dry weight, for sardine (1992-93).

\begin{tabular}{|lcccc|}
\hline Mes & $\mathrm{S}$ & $\mathrm{b}$ & $\mathrm{r} 2$ & $\mathrm{n}$ \\
\hline Abr & 390,3 & 4,533 & 0,71 & 186 \\
May & 396,1 & 4,236 & 0,57 & 312 \\
Jun & 434,2 & 4,501 & 0,84 & 263 \\
Jul & 415,8 & 5,312 & 0,81 & 291 \\
Ago & 428,8 & 4,979 & 0,93 & 269 \\
Sep & 422,0 & 4,852 & 0,89 & 229 \\
Oct & 390,0 & 5,040 & 0,80 & 207 \\
Nov & 366,2 & 5,426 & 0,81 & 173 \\
Dic & 370,3 & 5,928 & 0,88 & 128 \\
Ene & 418,3 & 4,209 & 0,92 & 176 \\
Feb & 418,4 & 4,368 & 0,89 & 174 \\
Mar & 393,6 & 4,942 & 0,89 & 189 \\
\hline
\end{tabular}

El parámetro K se presenta en la Fig. 1.

anual considerado no existen diferencias entre los estratos de talla para los valores estimados de PSUM y PSMI de estos meses ( $\mathrm{p}>0,05)$. 

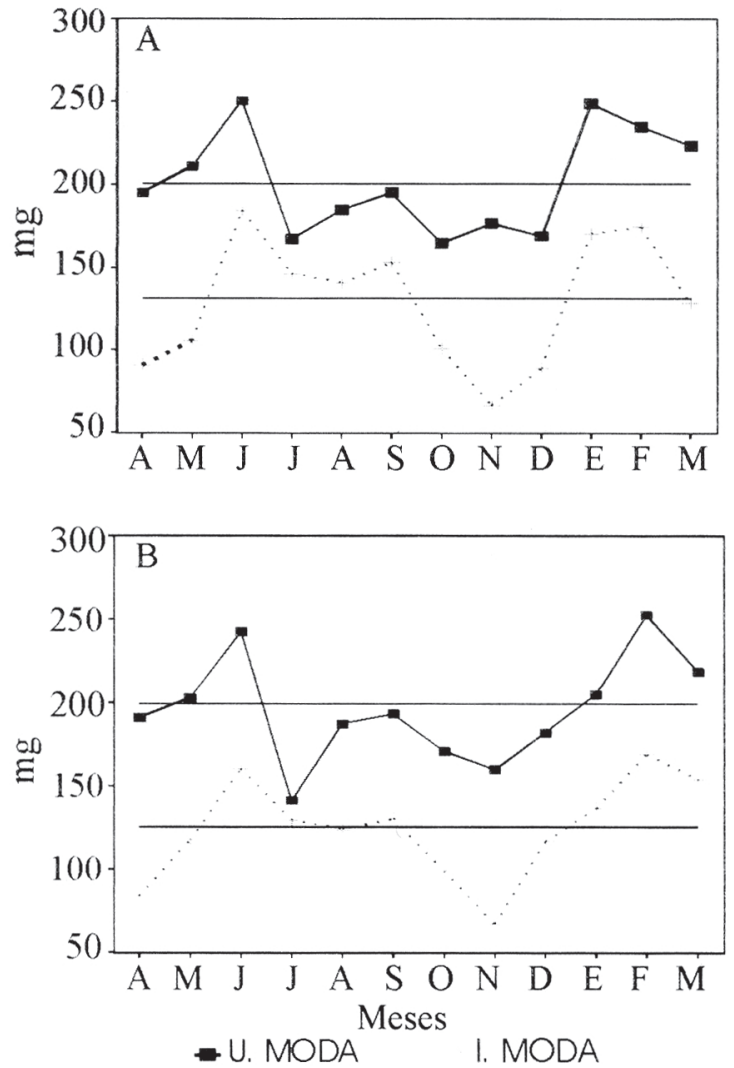

Figura 2. Peso seco estimado de la última moda (U. MODA) y de la moda intermedia (MODA I.) de ovocitos vitelados de sardina, en el rango de talla (A) $26-28 \mathrm{~cm}$ y (B) $28-30 \mathrm{~cm}$ de longitud total, y en estado de madurez sexual próximo a la hidratación. La línea horizontal corresponde a la media de todo el ciclo reproductivo.

Figure 2. Estimated last mode (L. MODE) and intermediate mode (I. MODE) dry weight of sardine yolked oocytes in $26-28 \mathrm{~cm}(\mathrm{~A})$ and $28-30 \mathrm{~cm}$ (B) total length strata in next to hydratation sexual maturity stage. The horizontal line is the average for the whole cycle.

\section{Peso seco del ovario}

Se encontraron diferencias significativas $(p<0,05)$ entre los promedio mensuales estimados del peso seco del ovario para los dos estratos de talla considerados. El promedio del peso seco estimado de la gónada para todo el ciclo anual correspondió a 4,05 g con una desviación estándar de $\pm 1,83$ g para el estrato de talla A y a 4,69 $\pm 2,10 \mathrm{~g}$ para el estrato de talla B; un máximo de $6,45 \pm 1,31$ g y un mínimo de

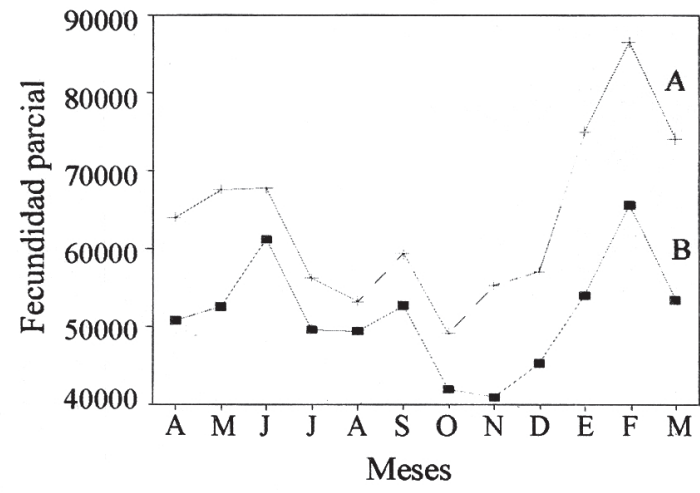

Figura 3. Fecundidad parcial de sardinas de $26-28 \mathrm{~cm}$ (A) y $28-30 \mathrm{~cm}$ (B), estandarizada a una hembra de peso corporal promedio de 180 g y 213 g respectivamente.

Figure 3. Batch fecundity of sardines in next to hydratation sexual maturity stage and in $26-28 \mathrm{~cm}$ (A) and 28-30 cm (B) total length strata.

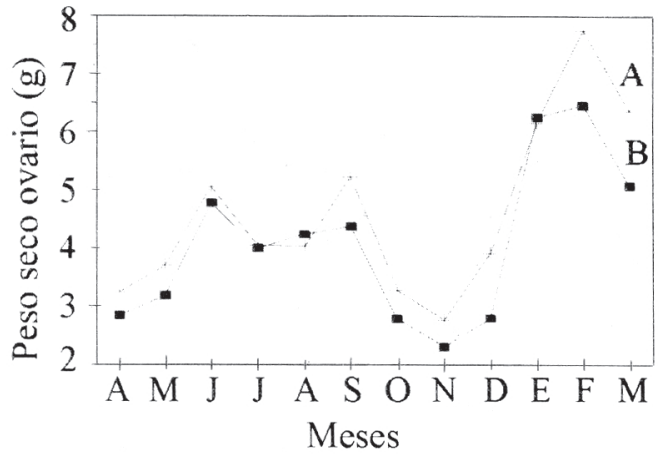

Figura 4. Peso seco estimado del ovario de sardinas en estado de madurez sexual próximo a la hidratación y en los rangos de talla $26-28 \mathrm{~cm}$ (A) y $28-30 \mathrm{~cm}$ (B).

Figure 4. Estimated ovary weight for sardines in next to hydratation sexual maturity stage and in $26-28 \mathrm{~cm}$ (A) and 28-30 cm (B) total length strata.

$2,29 \pm 0,47 \mathrm{~g}$ se estimaron para el estrato de talla A, y un máximo de 7,75 $\pm 2,34$ g y un mínimo de 2,75 $\pm 0,87$ g para el estrato de talla B (Fig. 4).

Durante el período reproductivo de invierno el peso seco promedio estimado del ovario del estrato de talla A se incrementó a partir de abril, hasta un máximo en junio $(\mathrm{p}<0,05)$; el valor de julio fue significativamente menor $(\mathrm{p}<0,05)$, y no presentó 
diferencias con el de agosto y septiembre ( $p>0,05)$. Por otro lado, no se encontró diferencias entre octubre, noviembre y diciembre $(\mathrm{p}>0,05)$, siendo estos valores significativamente menores a los de septiembre y enero.

Para el estrato de talla B se distingue un período de valores promedio estimados bajos de peso seco del ovario, que comprende los meses de octubre y noviembre, no existiendo diferencias entre los valores estimados para estos meses $(\mathrm{p}>0,05)$. A pesar de observarse valores relativamente bajos de peso seco del ovario para abril y mayo, éstos son mayores a los de octubre y noviembre $(\mathrm{p}<0,05)$, pero no difieren significativamente $(\mathrm{p}>0,05)$ de los promedios de julio y agosto.

Como resultado de este patrón en las variaciones del peso seco estimado del ovario para este estrato de talla, el período de desove de invierno se presenta con dos máximos que no presentan diferencias entre sí ( $\mathrm{p}>0,05)$, y que corresponden a junio y septiembre (Fig. 4).

El máximo valor alcanzado durante el período de desove de verano en ambos estratos de talla ocurre en febrero, el cual es significativamente mayor a los de enero y marzo $(\mathrm{p}<0,05)$. La diferencia entre los máximos de invierno y verano es muy marcada en ambos estratos de talla, y por tanto las diferencias entre febrero y junio son significativas para ambos estratos $(\mathrm{p}<0,05)$, como también lo es una comparación adicional para el estrato de talla B entre febrero y septiembre.

El valor promedio estimado del peso seco del ovario sigue en general para ambos estratos de talla el patrón de fecundidad parcial estimada para una hembra promedio (Fig. 3), siendo la principal diferencia entre ambos estratos la aparente recuperación en la fecundidad parcial en septiembre, luego de su baja en julio y agosto, lo que sólo se observa en las fluctuaciones del peso seco estimado de la gónada del estrato de talla B.

Una comparación entre estratos de talla de los valores promedio mensuales estimados para junio, julio, febrero, abril y noviembre, que corresponden a los valores máximos y mínimos para ambos estratos (Fig. 4), muestra que sólo existen diferencias de peso seco del ovario entre estratos en febrero, siendo el valor promedio del estrato de talla B para este mes significativamente mayor al estimado para el estrato de talla A $(\mathrm{p}<0,05)$.

\section{Condición de los desovadores}

\section{Estrato de talla A}

Se consideraron para el análisis de condición 267 hembras en estado de madurez sexual próximo a la hidratación (Claramunt et al., 1994), para las cuales se disponían registros mensuales de L. DOMA, fecundidad parcial, PSUM, PSMI y PSG. La variable fecundidad parcial fue eliminada debido a su alta correlación con las variables PSG $(r=0,78)$ y PSUM $(r=0,62)$. En un análisis posterior, para el que seleccionaron las cinco variables explicatorias restantes $(\mathrm{Cp}=4,0)$, se eliminaron del modelo de regresión las variables PSUM y PSMI (coeficientes $=-0,088$ y $-0,094$; valores $\mathrm{t}=-1,81$ y $-1,73$ respectivamente).

Los parámetros estimados para el modelo que incluye efectos mensuales (Ecuación 4), se indican en la Tabla 3. El modelo se comportó bien de acuerdo a los datos, presentando un $\mathrm{r}^{2}=0,75$ con una distribución normal y homocedástica de los residuos. El modelo lineal múltiple estimado y los coeficientes $\beta$ calculados (Tabla 4), implican que la condición de los desovadores en estado de madurez próximos a la hidratación para el estrato de talla A es explicada en un $78 \%$ por el modelo. El ln L explica un $52 \%$ de esta variación, el DOMA un 34\% y el PSG un $13 \%$.

Los 11 factores mensuales obtenidos, tomando enero de 1993 como base, se presentan en la Fig. 5. El vector de estos factores es una medida de la condición media en cada mes a través del ciclo anual 1992-93. La variación mensual en la condición de los desovantes muestra una declinación a partir de mayo, alcanzando un mínimo en junio y septiembre. Luego, hay un aumento en la condición que alcanza un máximo en diciembre, los meses de enero, febrero y marzo presentan una condición levemente inferior a diciembre.

\section{Estrato de talla $B$}

Se utilizó un total de 264 hembras maduras próximas a la hidratación, para las cuales se disponían registros de las variables ya señaladas para el estrato de talla A. En este caso la fecundidad parcial mostró un coeficiente de correlación de 0,8 con el PSG y de 0,56 con el PSUM. Debido a esto, y a que el PSG es una variable de mayor interés dado los objetivos de este estudio, la fecundidad parcial fue excluida de este análisis. El análisis de regresión de 
Tabla 3. Coeficientes del modelo In $W=\beta_{0}+\beta_{1}$ In $L$ $+\beta_{2}$ DOMA $+\beta_{3}$ PSG $+\gamma$ ajustado a ejemplares de sardina de $26-28 \mathrm{~cm}$ en estado de madurez sexual próximo a la hidratación.

Table 3. Coeficients for the model in $W=\beta_{0}+\beta_{1}$ In $L$ $+\beta_{2}$ DOMA $+\beta_{3}$ PSG $+\gamma$ ajusted using female sardines of $26-28 \mathrm{~cm}$ in next to hydratation sexual maturity stage.

\begin{tabular}{|lccc|}
\hline Variable & Coeficiente & Error Estándar & Valor t \\
\hline CONSTANTE & 0,5696 & 0,2062 & 2,7617 \\
ln L & 0,4058 & 0,0330 & 12,2810 \\
DOMA & $-0,0918$ & 0,0380 & $-2,4126$ \\
PSG & 0,1526 & 0,0452 & 3,3740 \\
\hline
\end{tabular}

265 observaciones; $\mathrm{r}^{2}=0,75$

Tabla 4. Coeficientes beta de las regresiones que explican las variaciones del peso corporal de sardina en los dos estratos de talla considerados (Tablas 3 y 5).

Table 4. Beta coeficients estimates for the models that explain the somatic weight variations of sardina in the two length strata considered (Tables 3 and 5).

\begin{tabular}{|cc|cc|}
\hline \multicolumn{2}{|c|}{ Estrato A } & \multicolumn{2}{c|}{ Estrato B } \\
\hline Variable & Coeficiente & Variable & Coeficiente \\
\hline In L & 0,5229 & In L & 0,4008 \\
DOMA & 0,3435 & PSUM & 0,2373 \\
PSG & 0,1336 & PSG & 0,3619 \\
\hline
\end{tabular}

Tabla 5. Coeficientes del modelo In $W=\beta_{0}+\beta_{1}$ In $L$ $+\beta_{2}$ PSUM $+\beta_{3}$ PSG $+\gamma$ ajustado a ejemplares de sardina de $28-30 \mathrm{~cm}$ en estado de madurez sexual próximo a la hidratación.

Table 5. Coeficients for the model $\ln W=\beta_{0}+\beta_{1} \ln L$ $+\beta_{2}$ PSUM $+\beta_{3}$ PSG $+\gamma$ using female sardines of 26$28 \mathrm{~cm}$ in next to hydratation sexual maturity stage.

\begin{tabular}{|lccr|}
\hline Variable & Coeficiente & Error Estándar & Valor $\mathbf{~}$ \\
\hline CONSTANTE & 0,5468 & 0,1256 & 4,3539 \\
ln L & 0,4896 & 0,0392 & 12,4947 \\
PSUM & $-0,1159$ & 0,0485 & $-2,3927$ \\
PSG & 0,2623 & 0,0576 & 4,5517 \\
\hline
\end{tabular}

264 observaciones; $\mathrm{r}^{2}=0,67$

las variables explicatorias restantes mostró que los coeficientes de las variables DOMA y PSMI (-0,074 $\mathrm{y}-0,11$; valores $\mathrm{t}=-1,54 \mathrm{y}-1,61$ respectivamente), no eran significativamente distintos de cero $(\mathrm{p}>0,05)$.

El modelo lineal que incluye variaciones estacionales en la condición, explica el $67 \%$ de la variación total con una distribución normal y homocedástica de los residuos. Los parámetros estimados se presentan en la Tabla 5; mientras que los coeficientes $\beta$ calculados se muestran en la Tabla 4.

La variación en la condición media mensual se muestra en la Fig. 5. En términos generales sigue la misma tendencia del estrato de talla A, presentando una baja condición de junio a septiembre, con un máximo en diciembre y una leve tendencia a declinar hacia enero-marzo.

\section{DISCUSIÓN}

Variación mensual del peso seco del ovario y modas de ovocitos vitelados

La cantidad de material seco contenida en las gónadas de sardina, de los dos estratos de talla considerados, indica fluctuaciones en la cantidad de energía destinada a la reproducción. Como es de esperar, tales fluctuaciones mantienen concordancia con los cambios intra-anuales de la cantidad de vitelo entregada al ovocito y en la fecundidad parcial (Fig. 1, 3 y 4), distinguiéndose los dos períodos principales de desove de la especie.

Los valores medios estimados del material seco de las dos modas de ovocitos vitelados representan la cantidad en mg contenida en un gramo húmedo de gónada (peso seco relativo). El peso seco relativo de la última moda de ovocitos fue el mismo para ambos períodos reproductivos (invierno y verano). Mientras que el peso seco relativo correspondiente a la moda intermedia es comparativamente menor a aquella de la última moda en cada estrato de talla. Sin embargo, entre estratos de talla la cantidad de material seco destinado por unidad de peso húmedo del ovario, tanto a la moda intermedia como a la última moda, es la misma entre períodos reproductivos correspondientes.

Esto significa que la energía relativa destinada a la reproducción sería la misma en ambos estratos de talla durante el ciclo anual considerado y que la energía relativa destinada al ovario en los máximos reproductivos de invierno y verano sería también de igual magnitud. No obstante, las hembras de mayor tamaño tienen también ovarios comparativamente más grandes (Claramunt et al., 1993) por lo que, en términos del total de la gónada, la energía 
destinada a la reproducción sería mayor en hembras de mayor tamaño. Esto último se observa en los valores promedio estimados para el peso seco del ovario, especialmente durante el período reproductivo de verano (Fig. 4).

\section{Condición de los desovadores}

Las propiedades de los factores de condición de peces, en la forma matemática de razones, hacen inválidos los análisis estadísticos paramétricos (Sokal y Rohlf, 1981). Además, frecuentemente tales factores se hallan correlacionados con la longitud del pez, permitiendo sólo la comparación entre poblaciones con distribuciones de longitud similares (Bolger y Connolly, 1989). Patterson (1992) propone un método para superar los problemas señalados, en donde todas las variables independientes que puedan afectar el peso son incluidas al mismo tiempo que la longitud, de modo de obtener una solución mínima cuadrada no sesgada. Para esto se utilizó una regresión de mínimos cuadrados, a pesar de que existen argumentos para utilizar una regresión funcional de media geométrica (MG) (Ricker, 1973). Sprent y dolby (1980) fide Cone (1989) destacan que el uso de regresiones de $\mathrm{MG}$ puede ser un tanto difícil e inapropiado y Cone (1989) recomienda el uso de una regresión común de mínimos cuadrados al trabajar con datos de longitud y peso.

Booth y Keast (1986) fide Bolger y Connolly (1989), han indicado que el crecimiento energético total puede teóricamente ocurrir independientemente de los cambios en peso corporal. Por esto, según Bolger y Connolly (1989) es necesario considerar con cuidado las interpretaciones en los cambios de condición medidos por índices basados sólo en datos de longitud y peso. En el presente estudio este análisis se efectuó paralelamente al registro de variables reproductivas, como son las fluctuaciones en peso seco de las fracciones de ovocitos vitelados de la gónada, la gónada total y el peso seco asintótico de los ovocitos de la moda más avanzada, contando además con mediciones de la fecundidad parcial y frecuencia de desove realizados por Claramunt et al. (1994), para este mismo ciclo anual. Todas estas variables representan fracciones de la energía destinada a la reproducción, cuyas fluctuaciones tienen una influencia importante sobre las reservas de energía de los desovadores (Kamler, 1992; Blaxter y Hunter, 1982).
Durante el ciclo 1992-93, se encontraron diferencias considerables en la condición de la sardina (Fig. 5). Los valores medios mensuales fueron bajos durante el período de desove de invierno, incrementándose a partir de septiembre y mostrando valores comparativamente altos para el máximo reproductivo de verano. La baja condición media en invierno coincide con la baja magnitud del peso seco estimado de la gónada y la caída en el peso seco de la última moda, moda intermedia y peso seco asintótico de los ovocitos en julio. Esta caída en los valores promedio también fue observada por Claramunt et al. (1994), en el tamaño del ovocito y la fecundidad.

En peces, la eficiencia de conversión de energía en crecimiento (crecimiento/ración de alimento) declina con la edad (Blaxter y Hunter, 1982), en tanto que la tasa metabólica declina exponencialmente con la talla (Winberg, 1961 fide Bradford y Stephenson, 1992) y el excedente energético disponible para un individuo se incrementa con la talla (Ware, 1984). Lo anterior explica lo señalado por De Martini (1991) para Seriphus politus, donde la condición somática varía con el tamaño corporal, de modo que hembras de mayor tamaño eran más robustas. Tal diferencia también fue encontrada para la sardina en el rango de talla considerado, donde los coeficientes de condición media siguen la misma tendencia para ambos estratos de talla, pero con valores comparativamente mayores para el estrato B (Fig. 5).

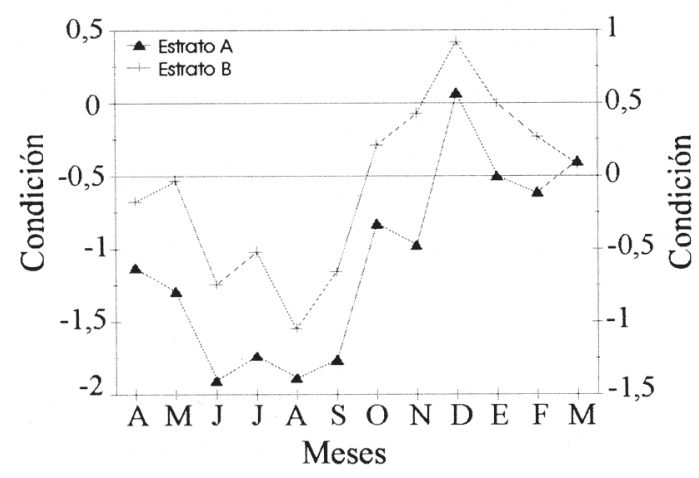

Figura 5. Condición mensual de hembras de sardina de $26-28 \mathrm{~cm}$ (A) y $28-30 \mathrm{~cm}$ (B) de longitud total.

Figure 5. Monthly condition estimates for sardine females in two total lenght strata $26-28 \mathrm{~cm}$ (A) and 28-30 cm (B). 
Los modelos ajustados incluyeron tres variables explicatorias. La longitud total y el PSG fueron variables comunes para ambos estratos de talla; como tercera variable se consideró el DOMA para el estrato A y el PSUM para el estrato B. La importancia relativa de las variables independientes consideradas, destaca el DOMA en relación a la condición de las hembras del estrato de talla A y al PSG en relación al estrato B (Tabla 4). Si se considera que las hembras de mayor talla presentan mayor fecundidad y períodos de desove más extensos e intensos, las variables asociadas a la condición media de las hembras en diferentes estratos de talla podrían indicar una mayor importancia relativa del tamaño del ovocito sobre la condición somática de las hembras de menor talla. Al incorporarse en el PSUM el número de ovocitos, la fecundidad tendría mayor importancia en hembras de tallas mayores.

\section{Temperatura y fluctuación mensual del contenido de energía del ovario}

Claramunt et al. (1994) consideran factible que la disminución en la fecundidad y la caída abrupta de ésta junto con la cantidad de vitelo del ovocito producida en julio, tenga relación con el fenómeno El Niño de 1991-92 (Fuenzalida, 1992). Los autores señalan que ésto podrían obedecer a una respuesta con retardo a la intrusión de aguas cálidas en el momento en que se estaban iniciando los procesos reproductivos correspondientes al invierno. Winters et al. (1993) y Tanasichuk y Ware (1987), señalan que las variaciones anuales en fecundidad serían probablemente afectadas por la temperatura de $2 \mathrm{o}$ 3 meses antes del desove.

No se intentó un análisis de correlación entre el peso seco de las modas y la temperatura, debido a que sólo se dispone de 12 estimaciones que comprenden el ciclo anual; el retardo mencionado en la respuesta reproductiva con respecto a la temperatura, implicaría un número menor de datos para un análisis de este tipo. Sin embargo, a continuación se discuten las posibles relaciones en base a referencias de la literatura.

Martínez et al. (1993) concluyen que para el desove de verano de 1992 (enero - febrero), la sardina presentó un alto procentaje de atresia y baja frecuencia de desove, implicando una disminución de la actividad reproductiva (en duración e intensidad) producto de El Niño 1991-92. Un evento El Niño, además de producir un calentamiento de las aguas superficiales, produce una profundización de la termoclina, reducción de las surgencias costeras y/o surgencias de aguas menos ricas en nutrientes, lo que conlleva a una disminución en la productividad primaria (Avaria, 1985a, 1985b).

A este respecto, es probable que los bajos valores de julio en el peso seco de las modas de ovocitos vitelados, como también en el peso seco estimado del ovario para el período de desove de invierno, se deban a una disminución en la disponibilidad de alimento potencial para los desovadores en los meses previos y durante el período de desove. Es sabido que una restricción en el alimento disminuye la fecundidad (Kamler, 1992) y puede causar tanto una disminución en la frecuencia del desove (Hunter y Leong, 1981), como una disminución en el tamaño del huevo (Tsuruta y Hirose, 1989). De Martini (1991) concluyó que la declinación observada en la fecundidad y condición somática de Seriphus politus durante 1984, reflejó una producción planctónica más baja durante el año más intenso de El Niño comparado con períodos más normales.

En el caso de la sardina, la condición media de los desovadores referida al peso somático (sin vísceras), muestra una baja evidente en el peso corporal medio (Fig. 5). Esto pudo deberse, además de la posible escasez de alimento, a la disminución de las reservas corporales producida por un esfuerzo reproductivo alto, ya que la fracción de hembras desovantes no evidenció ninguna baja en los meses correspondientes al desove principal (Claramunt et al., 1994).

Estos autores encontraron que la frecuencia de desove fue baja entre junio y octubre, período en el que se presentó el desove principal de invierno; esto coincide con lo reportado para la sardina por Martínez et al. (1993), para el mismo ciclo reproductivo, quienes concluyen que el desove principal de la especie mostró una disminución en intensidad, no obstante que la duración se mantuvo de acuerdo al patrón normal.

Wootton (1985) señala que los peces tienen la habilidad de controlar la partición de la energía entre reproducción y otros procesos vitales. Además, cualquier efecto de la limitación del alimento sobre la calidad del huevo está contrabalanceada por el hecho que un pez puede mantener la calidad del huevo a expensas de su número (Wootton, 1985), y los lípidos de las gónadas pueden ser usados para 
propósitos metabólicos bajo condiciones alimentarias extremas (Nikolskij, 1974 fide Kamler, 1992).

El conocimiento acerca de la alimentación de la especie es actualmente incompleto. No obstante, se conoce que Sardinops sagax es un filtrador omnívoro (Arancibia et al., 1986), que sus componentes tróficos principales los constituyen copépodos, diatomeas y dinoflagelados, y que la composición de especies en sus contenidos estomacales revela un espectro trófico amplio, con una composición volumétrica del plancton en su dieta dominada por fitoplancton en el área de Iquique, representando el zooplancton alrededor de un $23 \%$ del volumen estomacal. Herrera (1992a) reporta para marzo de 1992, que la importancia relativa de los dinoflagelados sobre las diatomeas fue clara, tanto en el número de especies como en los índices de abundancia relativa. Los dinoflagelados, especies típicas de aguas cálidas, son frecuentes en las muestras de verano, pero normalmente escasos en cantidad.

Fuenzalida (1992), encontró en julio evidencias térmicas cuasinormales que lo llevaron a concluir que el evento El Niño 1991-92 se encontraba en su etapa de finalización. Al respecto, el análisis cualitativo y cuantitativo realizado por Herrera (1992b) durante dicho mes, reflejó una comunidad fitoplanctónica característica de la zona norte de Chile, es decir con dominancia de diatomeas y altas concentraciones fitoplanctónicas a nivel costero producto de los eventos de surgencia (Avaria et al., 1982).

Como se explicó, el índice de condición se incrementa a partir de septiembre, mostrando valores comparativamente altos para el pico reproductivo de verano, lo que nuevamente nos da una diferencia de 2 a 3 meses entre el establecimiento de las condiciones térmicas en el área de estudio y el aumento de la condición de los desovadores y del peso seco de la gónada, el que como se indicó fue mayor durante el desove de verano que en el de invierno. Claramunt et al. (1994) encontraron además que la fracción de hembras desovante fue mayor en verano; situación interesante, ya que ésta ha sido considerado como un pico de desove secundario en la sardina (Martínez et al., 1990, 1993), lo que a criterio de estos autores podría constituir una respuesta compensatoria al fenómeno El Niño 1991-92. Martínez et al. (1989) reportan que la sardina desovó con mayor intensidad durante 1984 , en respuesta al fenómeno El Niño 1982-83; durante este año no se observó la etapa de reposo que normalmente se presenta entre abril y mayo, manifestándose desoves significativos durante casi todo el año (Retamales y González, 1985).

No obstante lo anterior, los datos obtenidos y los antecedentes disponibles no permiten establecer si una limitación en el alimento es el o uno de los factores causales de los bajos valores en determinadas variables reproductivas. Vernberg y Vernberg (1972) fide Sarojini y Gyananath (1985) señalan que otros factores medioambientales, además del alimento, interactuan con factores endógenos para iniciar e influir la naturaleza y el patrón de la reproducción, y el comportamiento reproductivo en vertebrados.

La temperatura sería el principal factor regulador del tamaño del huevo (Daoulas y Economou, 1986; De Martini, 1991). Claramunt et al. (1994) muestran que para la sardina esto podría ser válido inter-estacionalmente. Ha sido sugerido por Tanasichuk y Ware (1987), que la base biológica de ésta regulación del tamaño del ovocito es la atresia pre-ovulatoria (involución o reclutamiento de ovocitos; Wallace y Selman, 1981). A su vez, este proceso es regulado por la temperatura del mar que modificaría los niveles plasmáticos de gonadotropinas durante la vitelogénesis (Billard et al., 1982). Hunter y Macewicz (1985) encontraron que Engraulis mordax puede reabsorber rápidamente (en algunos meses o menos) los ovarios ante una eventual escasez de alimento, sin dejar ninguna evidencia de actividad reproductiva. Sin embargo, las tasas de atresia en sardina no son conocidas, por lo que no se sabe si ésta es capaz de explicar la caída en la fecundidad y tamaño del ovocito en la escala de tiempo en que se presentó durante la estación reproductiva estudiada. Estos autores indican también, que dada una ración de alimento suficiente la atresia se detiene, y la maduración y la vitelogenésis es reasumida, produciéndose un ovario reproductivamente activo en un período de 35 días.

La cantidad de energía destinada a la reproducción en la sardina parece variar de acuerdo a la fluctuación de las condiciones ambientales. Sin embargo, no se pueden precisar las variables biológicas y ambientales que explican esta respuesta en la especie, debido a la escasa resolución en la información ecológica para el ciclo anual considerado, desconociéndose además la posible relación funcional entre estas variaciones energéticas y dichas variables 
ambientales. Responder a la pregunta formulada por Claramunt et al. (1994), acerca de la canalización de la energía, requiere de una base de datos más extensa, que comprenda tanto información reproductiva como ambiental. Además implica el conocimiento tanto de tasas de crecimiento como para el ciclo anual estudiado. Blaxter y Hunter (1982) señalan que la mayoría de la energía en peces adultos parece ser empleada en el metabolismo, con la reproducción y el crecimiento ubicados en segundo lugar de importancia.

No obstante la carencia de información acerca de las variables ambientales de interés, como de la continuidad en el tiempo de la información requerida para aclarar nuestro conocimiento sobre la estrategia reproductiva de la especie, se pueden señalar las siguientes conclusiones:

- la sardina muestra fluctuaciones en la cantidad de energía destinada a reproducción, las cuales concuerdan con los cambios intra-anuales del tamaño medio del ovocito de la moda más avanzada y de la fecundidad parcial.

- las fluctuaciones intra-anuales de la cantidad de energía destinada a la moda más avanzada y a la moda intermedia de ovocitos presentan un patrón similar, coincidiendo sus máximos y mínimos; en otras palabras, el proceso de vitelación en curso tiende a alcanzar el valor del peso seco de la moda ya conformada.

- la energía relativa (cantidad de vitelo por unidad de peso húmedo de la gónada) destinada a reproducción, no varía con la longitud del pez a través de la totalidad del ciclo anual, para el rango de talla considerado.

- la energía relativa destinada al ovario es la misma en ambos períodos reproductivos (invierno y verano).

- la cantidad de energía destinada al ovario fue mayor en el verano que en invierno; en otras palabras, si bien la cantidad de vitelo por unidad de peso húmedo del ovario fue la misma en ambas estaciones, los ovarios presentaron mayor peso total en verano; esto correspondería a una respuesta compensatoria al bajo número y tamaño de los ovocitos desovados en invierno.

\section{REFERENCIAS}

Alheit, J. 1988. Reproductive Biology of sprat (Sparattus sprattus): factors determining annual egg production. J. Cons. Int. Explor. Mer, 44: 162-168.

Arancibia, H., E. Oliva y J. Braun. 1986. Alimentación de la sardina española (Sardinops sagax) de la zona norte de Iquique en el período noviembre ' 85 - noviembre '86. Inf. Proy. Invest. IFOP-UNAP, $33 \mathrm{pp}$.

Avaria, S., P. Muñoz y E. Uribe. 1982. Composición y biomasa del fitoplancton marino del norte de Chile en diciembre de 1980 (MARCHILE XI-ERFEN III). Cienc. Tec. Mar, CONA 7: 109-140.

Avaria, S. 1985a. Efectos de El Niño en las pesquerías del Pacífico Sureste. Invest. Pesq. (Chile), 32: 101-116.

Avaria, S. 1985b. Variaciones en la composición y biomasa del fitoplancton marino del norte de Chile entre diciembre 1980 y junio 1984. Invest. Pesq. (Chile), 32: 191-193.

Billard, R., A. Fostier., C. Weil y B. Breton. 1982. Endocrin control of spermatogenesis in teleost fish. Can. J. Fish. Aquat. Sci., 39: 65-79.

Blaxter, J.H. y G. Hempel. 1963. The influence of egg size on herring larvae (Clupea harengus L.). J. Cons. Int. Explor. Mer, 28: 211-240.

Blaxter, J.H. y J.R. Hunter. 1982. The biology of the clupeioid fishes. Adv. Mar. Biol., 20: 1-223.

Bolger, T. y P. Connolly. 1989. The selection of suitable indices for measurement and analysis of fish condition. J. Fish. Biol., 34: 171-182.

Bradford, R.G. y R.L. Stephenson. 1992. Egg weight, fecundity, and gonad weight variability among north-west Atlantic herring (Clupea harengus) populations. Can. J. Fish. Aquat. Sci., 49: 20452054.

Canavos, G. 1987. Probabilidad y estadística. McGraw - Hill, México, 651 pp.

Chatterjee, S. y B. Price. 1977. Regression analysis, Wiley \& Sons., New York, 228 pp. 
Claramunt, G., G. Herrera y P. Pizarro. 1993. Fluctuaciones de la fecundidad parcial en sardina española (Sardinops sagax) durante la época principal de desove de 1990, en la zona norte de Chile. Sci. Mar., 57(1): 9-14.

Claramunt, G., G. Herrera y P. Pizarro. 1994. Producción potencial anual de huevos por tallas en Sardinops sagax (Jenyns, 1982) del norte de Chile. Rev. Biol. Mar., Valparaíso, 29(2): 211-233.

Clarke, T.A. 1989. Seasonal differences in spawning, egg size, and early development time of the hawaiian anchovy or nehu, Encrasicholina purpurea. Fish. Bull., U.S., 87(3): 593-600.

Cone, R.C. 1989. The need to reconsider the use of condition indices in fishery science. Trans. Am. Fish. Soc., 118: 510-514.

Conover, W.J. 1980. Practical nonparametric statistics. Wiley \& Sons., New York, 493 pp.

Cushing, D.H. 1990. Plankton production and yearclass strength in fish populations: an update of the match/mismatch hypothesis. Adv. Mar. Biol., 26: 249-293.

Daoulas, C.H. y A. Economou. 1986. Seasonal variation of egg size in the sardine, Sardina pilchardus Walb., of the Saronikos Gulf: causes and a probable explanation. J. Fish. Biol., 28: 449-457.

De Martini, E. 1991 Annual variations in fecundity, egg size, and the gonadal and somatic conditions of queenfish Seriphus politus (Sciaenidae). Fish. Bull., U.S., 89: 9-18.

Dumont, S.N. y A.R. Brummett. 1985. Egg envelopes in invertebrates. In: Development biology. Leon W. Brower (Ed.), (1): 235-277.

Fuenzalida, R. 1992. Oceanografía física. In: Monitoreo de las condiciones bio-oceanográficas en la zona norte de Chile (18 18'S-2331'S). Prog. INPESCON 1992, Univ. Arturo Prat, Dep. Ciencias del Mar, Iquique - Chile. Doc. Téc., 52: 5-13.

Hempel, G. 1979. Early life history of marine fish: the egg stage. Univ. Washington Press, Seattle, 70 pp.

Hempel, G. y J.H.S. Blaxter. 1967. Egg weight in Atlantic herring (Clupea harengus L.). J. Cons. Int. Explor. Mer, 31: 170-195.

Herrera G. y G. Claramunt. 1990. Estimaciones de la fecundidad parcial y frecuencia de desove de Sardinops sagax durante 1987-88, en el norte de Chile. Invest. Cient. y Tec., Serie Ciencias del Mar, 1: 55-68.

Herrera, L. 1992a. Fitoplancton. In: Monitoreo de las condiciones bio-oceanográficas en la zona norte de Chile (18¹8'S-2331'S). Prog. INPESCON 1992, Univ. Arturo Prat, Dep. Ciencias del Mar, Iquique - Chile. Doc. Téc., 46C: 20-32.

Herrera, L. 1992b. Fitoplancton. In: Monitoreo de las condiciones bio-oceanográficas en la zona norte de Chile (18¹8'S-23³1'S). Prog. INPESCON 1992, Univ. Arturo Prat, Dep. Ciencias del Mar, Iquique - Chile. Doc. Téc., 52: 22-38.

Hunter, J.R. 1981. Feeding ecology and predation of marine fish larvae. In: Marine fish larvae. R. Lasker (Ed.), Univ. Washington Press, Seattle, pp. 33-77.

Hunter, J.R. y R. Leong. 1981. The spawning energetics of female northern anchovy Engraulis mordax. Fish. Bull., U.S., 79: 215-230.

Hunter, J.R. y B.J. Macewicz. 1985. Measurement of spawning frequency in multiple spawning fishes. In: An egg production method for estimating spawning biomass of pelagic fish: application to the northern anchovy, Engraulis mordax. R. Lasker (Ed.), NOAA-NMFS, Tech. Rep., 36: 79-94.

Imai, C. y S. Tanaka. 1987. Effect of sea water temperature on egg size of japanese anchovy. Nippon Suisan Gakkaishi, 53(12): 2169-2178.

Johnson, R.A. y D.W. Wichern. 1982. Applied multivariate statistical analysis. Prentice - Hall, New Yersey, 607 pp.

Kamler, E. 1992. Early life history of fish an energetics approach. I. Winfield \& S. Nelson (Eds.), Chapman \& Hall, London, 267 pp.

Kjesbu, O.S. 1989. The spawning activity of cod, Gadus morhua. J. Fish. Biol., 34: 195-206.

Knutsen, G.M. y S. Tilseth. 1985. Growth, development, and feeding success of Atlantic cod larvae Gadus morhua related to egg size. Trans. Am. Fish. Soc., 114: 507-511.

Le Clus, F. 1979. Dry mass of yolked oocytes of the 
south west african pilchard Sardinops ocellata in relation to maturity stages spawning cycles, 197274. Invest. Rep. Sea Fish., Branch South Africa, 119: $1-29$.

Le Clus, F. 1992. Seasonal trends in sea surface temperature, dry mass per oocyte and batch fecundity of pilchard Sardinops ocellatus in the Northern benguela system. S. Afr. J. Mar. Sci., 12: 123-134.

Macewicz, J. y R. Hunter. 1993. Spawning and batch fecundity of jack mackerel, Tranchurus symmetricus, off California during 1991. Calif. Coop. Oceanic Fish. Invest. Rep., 34: 112-121.

Manugistics. 1992. Stratigraphics reference manual. Version 6.0. Statistical graphics corporation, Maryland.

Marquardt, D.W. 1963. An algorithm for least-squares estimation of nonlinear parameters. J. Soc. Ind. App. Math., 11: 431-441.

Martínez, C., L. Caballero, G. Böhm, A. Torres, R. Gili, V. Bocic, H. Muñoz, P. Barría y R. Serra. 1989. Diagnóstico de las principales pesquerías pelágicas de la zona norte (I-IV Región, 1988). CORFO-IFOP, Santiago - Chile, AP 89/17c, 72 pp.

Martínez, C., L. Caballero, G. Böhm, R. Gili, V. Bocic, P. Barría y R. Serra. 1990. Diagnóstico de las principales pesquerías pelágicas de la zona norte (I-IV Región, 1989). CORFO-IFOP, Santiago Chile, AP 90/10, 119 pp.

Martínez, C., U. Parker, L. Caballero, G. Böhm, J. Oliva, S. Peña, R. Gili, H. Hidalgo, V. Fernández, P. Barría, R. Serra y J. Blanco. 1993. Diagnóstico de las principales perquerías pelágicas de la zona norte (I - IV Región, 1992). CORFO-IFOP, Santiago - Chile, SGI-IFOP 93/6, 134 pp.

Miller, T.J., L.B. Crowder, J.A. Rice y E.A. Marschall. 1988. Larval size and recruitment machanisms in fishes: towards a conceptual framework. Can. J. Fish. Aquat. Sci., 45: 16571670.

Patterson, K.R. 1992. An improved method for studying the condition of fish, with an example using pacific sardine Sardinops sagax (Jenyns). J. Fish. Biol., 40: 821-831.

Pindyck, R. y D. Rubinfeld. 1980. Modelos econométricos. MsGraw - Hill, Barcelona, 638 pp.
Pizarro, P. 1990. Influencia de la estructura de tallas en la época y longitud de desove de sardina española (Sardinops sagax) en la zona norte de Chile. Tesis Biól. Pesq., Univ. Arturo Prat, Dep. Ciencias del Mar, Iquique - Chile, 82 pp.

Retamales, R y L. González. 1985. Incidencia del fenómeno de El Niño 1982-83 en el desove de sardina española (Sardinops sagax). Invest. Pesq. (Chile). 32: 161-165.

Ricker, W.E. 1973. Linear regressions in fisheries research. J. Fish. Board Can., 30: 409-434.

Sarojini, R. y G. Gyananath. 1985. Photoperiodic regulation of ovarian maturation in the freshwater prawn Macrobrachium lamerri. Curr. Biosci., 2(4): 158-162.

Simpson, A.C. 1951. The fecundity of the plaice. Fish. Invest. London Ser., 17(5): 27 pp.

Sokal, R.R. y F.J. Rohlf. 1981. Biometry. W.H. Freeman Co., San Francisco, 776 pp.

Steel, R.G. y J.H. Torrie. 1985. Bioestadística. McGraww - Hill, Colombia, 622 pp.

Tanasichuk, R.W. y D.M. Ware. 1987. Influence of interannual variations in winter sea temperature on fecundity and egg size in pacific herring (Clupea harengus pallas). Can. J. Fish. Aquat. Sci., 44: 1485-1495.

Tsuruta, Y y K. Hirose. 1989. Internal regulation of reproduction in the Japanese anchovy (Engraulis japonica) as related to population fluctuation. In: Effects of ocean variability on recruitment and an evaluation of parameters used in stock assessment models. R.J. Beamish \& G.A. McFarlane (Eds.), Can. Spec. Publ. Fish. Aquat. Sci., 108: 111-119.

Wallace, R. y K. Selman. 1981. Cellular and dynamic aspects of oocyte growth in teleosts. Amer. Zool., 21: 325-343.

Ware, D.M. 1975. Relation between egg size, growth and natural mortality of larval fish. J. Fish. Res. Board Can., 32: 2503-2512.

Ware, D.M. 1977. Spawning time and egg size of atlantic mackerel, Scomber scombrus, in relation to the Plankton. J. Fish. Res. Board Can., 34: 23082315 .

Ware, D.M. 1984. Fitness of different reproductive strategies in teleost fishes. In: Fish reproduction, 
G. W. Pots \& R.J. Wootton (Eds.), Academic Press, New York, pp. 349-366.

Winters, G.H., J.P. Wheeler y D. Stansbury. 1993. Variability in the reproductive output of springspawning herring in the north-west Atlantic. ICES, J. Mar. Sci., 50: 15-25.

Webb, P.W. y D. Weihs. 1986. Functional locomotor morphology of early life history stages of fishes. Trans. Am. Fish. Soc., 115: 115-127.
Weibull, W. 1951. A statistical distribution function of wide applicability. J. Appl. Mechanics, 18: 293297.

Wootton, R.J. 1985. Energetics of reproduction. In: Fish energetics: new perspectives, P. Tyler \& P. Calow (Eds.), Croom Helm, London, pp. 231-254.

Zar, J.H. 1984. Biostatistical analysis. Prentice-Hall, Englewood Cliffs, New Jersey, 718 pp.

Recibido el 18 de octubre de 1995.

Aceptado el 11 de noviembre de 1996. 